\title{
BMJ Open Use of compression therapy to treat lower limb wounds across Europe: a scoping review protocol
}

\author{
Ray Samuriwo (1) , ${ }^{1,2}$ Natalia Christiansen, ${ }^{3}$ Alison Hopkins ${ }^{4}$
}

\begin{abstract}
To cite: Samuriwo R, Christiansen N, Hopkins A. Use of compression therapy to treat lower limb wounds across Europe: a scoping review protocol. BMJ Open 2020;10:e039008. doi:10.1136/ bmjopen-2020-039008

- Prepublication history for this paper is available online. To view these files, please visit the journal online (http://dx.doi. org/10.1136/bmjopen-2020039008).
\end{abstract}

Received 31 March 2020 Revised 20 May 2020 Accepted 03 August 2020

Check for updates

(C) Author(s) (or their employer(s)) 2020. Re-use permitted under CC BY-NC. No commercial re-use. See rights and permissions. Published by BMJ.

${ }^{1}$ School of Healthcare Sciences, Cardiff University College of Biomedical and Life Sciences, Cardiff, UK

${ }^{2}$ Wales Centre for Evidence Based Care, School of Healthcare Sciences, Cardiff University, Cardiff, Wales, United Kingdom

${ }^{3}$ European Wound Management Association, Frederiksberg, Denmark

${ }^{4}$ Accelerate CIC, St Joseph's Hospice, London, UK

Correspondence to Dr Ray Samuriwo; samuriwor@cardiff.ac.uk

\begin{abstract}
Introduction Poor lower wound care is an avoidable patient harm. Compression therapy is an effective way of treating non-ischaemic lower limbs wounds, but it is not always used appropriately. There are many guidelines which set out how compression therapy should be used, but there is dearth of evidence about how it is actually used at a population level across Europe.

Aim The aim of this scoping review is to map the evidence published in English relating to the use of compression therapy to treat lower limb wounds across Europe.

Methods This scoping review will be conducted in line with the Joanna Briggs Institute and Preferred Reporting Items for Systematic Reviews and Meta-Analyses for Protocols and Scoping Reviews guidance. A search for relevant publications will be conducted on variety of databases and key websites in order to identify a comprehensive range of relevant literature. Peer reviewed empirical papers, theoretical papers and other publications in English relating to the use of compression therapy across Europe will be considered for inclusion.

Ethics and dissemination Ethical and research governance for this scoping review is not required because we will only gather secondary data. Our results will be disseminated to the widest possible audience through an open access paper in a peer reviewed international journal, conference presentations and a plain English summary. The results of this scoping review will be used by a panel of Key Opinion Leaders from across Europe to develop a driver diagram to underpin subsequent lower limb wound care improvement efforts.
\end{abstract}

\section{INTRODUCTION}

Poor lower limb wound care is an avoidable patient harm which has an adverse impact on a patient's quality of life and healthcare related outcomes. ${ }^{12}$ One aspect of lower limb wound care that can be improved is the appropriate use of compression therapy. ${ }^{2}$ There are a variety of guidelines ${ }^{3-5}$ which explain how compression therapy should be used to treat non-ischaemic lower limb wounds. However, there have been some reports ${ }^{67}$ of the inappropriate use of compression therapy to treat venous leg ulcers and diabetic foot ulcers. There are also some anecdotal reports ${ }^{12}$ of

\section{Strengths and limitations of this study}

- This protocol sets out the first scoping review that will systematically map evidence published in English relating to the use of compression therapy for lower limb wound healing across Europe.

- This scoping review will use a detailed search strategy designed to identify a comprehensive range of published peer reviewed publications and grey literature in English.

- We will adhere to the best practice in conducting scoping reviews as set out by the Joanna Briggs Institute and the Preferred Reporting Items for Systematic Reviews and Meta-Analyses for Scoping Reviews guidance.

- Only studies published in English will be included and therefore, this scoping review will provide an insight into what is published in English about use of compression in Europe, but will not establish the actuality of use because it will not include non-English language publications.

- The quality of evidence that we retrieve will not be assessed as this is a scoping review.

compression therapy not being used to treat lower limb wounds for a variety of reasons including the view that patients refuse to have or cannot tolerate this treatment. The reported issues in the use of compression therapy contravene the global focus on universal health coverage ${ }^{8}$ that improves population health and fosters the sustainable development of nations. Universal health coverage can only be achieved through the delivery of safe, fair, just affordable and equitable care. ${ }^{8}$ Therefore, it is it is vital that evidence-based compression therapy related improvement interventions are implemented to achieve lower limb wound care related universal coverage by reducing unwarranted variation.

Healthcare is delivered in systems that are complex and adaptive, therefore it is vital that quality improvement efforts are informed by theory and designed to improve practice at a system level. ${ }^{9} 10$ The patient trajectory in 
healthcare is such that there are some aspects of care delivery which are uncertain and emergent. ${ }^{10-12}$ Healthcare is delivered in a pressurised context with a complex adaptive ecology ${ }^{13-15}$ with emergent, uncertain elements arising from the contingencies of clinical practice ${ }^{12}$ which can be codified, convoluted or concatenated. Consequently, healthcare delivery is predicated on a combination of formal processes and structures such as policies as well as negotiations and adaptation throughout the patient care trajectory. ${ }^{1012}$ Emergent aspects of healthcare organisation often exist outside of formal management structures and tend to be overlooked in managerial narratives and improvement efforts. ${ }^{10}{ }^{12}$ Emergent aspects of healthcare are often tacit and are sometimes referred to as 'fugitive knowledge' or 'soft intelligence' because they exist outside of formal knowledge systems and structures. ${ }^{16}$

Healthcare systems are inherently fractal and selfsimilar, so improvement efforts need to be cognisant of individual, social and cultural factors that are at play in any given context. ${ }^{1718}$ There is also an emerging consensus that healthcare improvement efforts can only be effective when there is due awareness and recognition of the emergent, negotiated and tacit aspects that are inherent in the complexity of clinical practice..$^{10}$ The most effective quality improvement interventions provide a set of principles that can be adapted and used to improve the quality of care in different contexts. ${ }^{9}{ }^{10}$ Given the harm that can be caused by poor lower limb wound care, there is an urgent need to identify the best available empirical evidence, relevant theory and other publications that can be used at a system level to improve the use of compression therapy in different contexts. It is important to scope the theoretical evidence and other evidence such as opinion papers on compression therapy given the emergent aspects of healthcare systems which are often tacit and are less likely to be established through formal means such as empirical studies.

\section{AIM}

There is a dearth of evidence on the use of compression therapy at a population level from across Europe. We intend to address this gap in wider literature in this scoping review by mapping the English language publications relating to the use of compression therapy in lower limb wound healing. In order to achieve this aim, we intend to answer five related research questions (RQs), which are yet to be addressed in wider literature about compression therapy that is:

RQ1: what is the evidence published in English about the use of high compression therapy and reduced compression therapy in lower limb wound healing across Europe?

RQ2: what factors are reported to influence the use of high compression therapy and reduced compression therapy in lower limb wound healing across Europe?
RQ3: what guidance is used to inform the use of high compression therapy and reduced compression therapy in lower limb wound healing across Europe?

RQ4: what are the barriers and facilitators related to the appropriate use of high compression therapy and reduced compression therapy in lower limb wound healing across Europe?

RQ5: what if any narratives about the use of high compression therapy and reduced compression therapy influence how they are used in patient care?

\section{METHODS}

\section{Design}

We will undertake a scoping review in line with Joanna Briggs Institute (JBI) methods ${ }^{19}$ as well as Preferred Reporting Items for Systematic Reviews and Meta-Analyses (PRISMA) and Scoping Reviews guidance. ${ }^{20}$

\section{Search strategy and information sources}

A three-step search for relevant published and unpublished literature will be conducted according the JBI methods. ${ }^{21}$ The initial search will be undertaken on PubMed and CINAHL to identify key search terms and Medical Subject Headings that can be used to obtain literature on the use, misuse or non-use of compression therapy to treat lower limb wounds. In the second stage of the literature search, our strategy and search terms will be adapted for each database or website. In this stage, we will search for relevant literature on EMBASE, Applied Social Sciences Index and Abstracts, Web of Science, Turning Research into Practice, the Cochrane library and the JBI database of systematic reviews and evidence syntheses. We will also search for relevant literature on key sites including Google Scholar, Ethos, OpenGrey, ProQuest Dissertations and Theses, in addition to hand searching the latest editions of relevant journals. In the final stage of the search, we will undertake back and forward chaining in order to identify any additional published or unpublished literature.

\section{Screening and selection}

All of retrieved citations will be exported to the review software Rayyan, ${ }^{22}$ in which any duplicates will be removed. The titles and abstracts of the remaining citations will be independently assessed against the inclusion criteria by two members of the project team (RS and NC). In cases where there is doubt about whether to consider a paper for inclusion, the full text of that publication will be retrieved. Any differences of opinion about the inclusion of a paper in this scoping review will be resolved by an independent third reviewer $(\mathrm{AH})$.

\section{Inclusion and exclusion criteria}

We will include published and unpublished literature on compression therapy from 1990 onwards in this scoping review. The first European studies and guidelines on the use of compression therapy were published were 
Table 1 Inclusion criteria

\begin{tabular}{|c|c|c|c|}
\hline Population & Concept & Context & Type of studies and papers \\
\hline $\begin{array}{l}\text { Adults with lower limb } \\
\text { wounds in Europe }\end{array}$ & $\begin{array}{l}\text { Compression therapy or } \\
\text { high compression therapy } \\
\text { or reduced compression } \\
\text { therapy }\end{array}$ & $\begin{array}{l}\text { Hospital or secondary care } \\
\text { or tertiary care or community } \\
\text { or primary care or care } \\
\text { home or nursing home or } \\
\text { residential home or sheltered } \\
\text { accommodation or hospice }\end{array}$ & $\begin{array}{l}\text { Qualitative studies, quantitative } \\
\text { studies, mixed method studies, } \\
\text { systematic reviews, quality } \\
\text { improvement projects, audits, } \\
\text { discussion papers, editorials } \\
\text { guidelines, consensus statements, } \\
\text { policies, case studies, opinion } \\
\text { papers }\end{array}$ \\
\hline
\end{tabular}

published in the $1990 \mathrm{~s},{ }^{23-26}$ so we will search for literature published from the start of this decade. Only literature published in English will be considered for inclusion as there is no facility for translation. Our inclusion criteria for this scoping review are summarised in table 1 .

\section{Data extraction}

We have developed a specially designed data extraction table for this scoping review to gather relevant information from each publication (see online supplemental appendix 1). As per JBI guidance, ${ }^{19}$ this data extraction tool maybe further refined and developed as the scoping review progresses. The authors of any publications with missing data will be conducted for further information by the project team where possible. In line with the principles of a scoping review, data will be extracted from all included publications irrespective of their quality. The data extraction will be undertaken by two members of the project team (RS and NC) to ensure that relevant data are extracted in appropriate manner. Any differences of opinion will be recorded and resolved by the third reviewer $(\mathrm{AH})$.

\section{Data presentation}

The process by which publications are selected for inclusion in this scoping review will be summarised in a PRISMA flowchart. ${ }^{19}$ Pertinent data from included publications will be tabulated in relation key concepts in the inclusion criteria, that is, population, concept and context. Data will be tabulated and presented in a narrative format with accompanying text describing the relationship to the review questions.

\section{PATIENT AND PUBLIC INVOLVEMENT}

The development and design of this scoping review protocol was not directly informed by patients or members of the public given our focus the use of compression therapy as an intervention to promote lower limb wound healing. However, the quality and safety of patient care with regard to the use of compression therapy to promote wound healing in the lower limb is a key focus in this scoping review.

\section{ETHICS AND DISSEMINATION}

Ethical and research governance approval is not required as we will be conducting a scoping review that will not gather any primary data. To the best of our knowledge, this is the first scoping review that has sought to establish what is known about the use of compression therapy for lower limb wound healing across Europe. Our scoping review will map what is known about the use, misuse or non-use of compression therapy to promote lower limb wound healing across Europe. Consequently, the results of this scoping review will be of interest to an international audience of healthcare professionals who are keen to improve the quality of lower limb wound care at a population level.

We intend to share the results of this scoping review with the widest possible audience. The results of this study will be disseminated via conference presentations, a peer-reviewed open access international journal publication and a report. A plain English summary of our results will also be prepared so that it can be translated and disseminated to people with lower limb wounds and their families across Europe. Our results will also be shared online via the European Wound Management Association website. We also intend for the findings of this scoping review to be used to inform efforts to improve the use of compression therapy in order to deliver the best possible patient outcomes across Europe. To this end, we will host a meeting of an interprofessional pan European Key Opinion Leaders at the next European Management Wound Association conference to discuss our results in relation to expert opinion, and to identify key change concepts that can be integrated into a programme theory, in the form of a driver diagram, ${ }^{27} 28$ to underpin future improvement initiatives.

Acknowledgements We would like to thank the European Wound Management Association for supporting this scoping review. We would also like to acknowledge with thanks, Essity, Hartmann and Urgo Medical for funding the European Wound Management Association compression therapy quality improvement project through an unrestricted educational grant.

Contributors The scoping review was conceived and designed by RS. This study will be undertaken by RS, NC and AH. Data collected by RS, NC. Data analysis and interpretation conducted by $\mathrm{RS}, \mathrm{NC}$ and $\mathrm{AH}$. First draft of manuscript was written by RS. Authors who contributed to the writing of the manuscript: RS, NC and AH. ICMJE criteria for authorship met by: RS, NC and AH. Read and approved the manuscript as submitted: RS, NC and $\mathrm{AH}$. 
Funding This scoping review is a part of the European Wound Management Association compression therapy quality improvement project. This compression therapy quality improvement project is funded through an unrestricted educational grant from Essity, Hartmann and Urgo Medical.

Competing interests One of the authors, NC is employed by the European Wound Management Association.

Patient and public involvement Patients and/or the public were not involved in the design, or conduct, or reporting, or dissemination plans of this research.

Patient consent for publication Not required.

Provenance and peer review Not commissioned; externally peer reviewed.

Open access This is an open access article distributed in accordance with the Creative Commons Attribution Non Commercial (CC BY-NC 4.0) license, which permits others to distribute, remix, adapt, build upon this work non-commercially, and license their derivative works on different terms, provided the original work is properly cited, appropriate credit is given, any changes made indicated, and the use is non-commercial. See: http://creativecommons.org/licenses/by-nc/4.0/.

ORCID iD

Ray Samuriwo http://orcid.org/0000-0001-5954-0501

\section{REFERENCES}

1 Hopkins A. Queens nursing Institute blog, 2018. Available: https:// www.qni.org.uk/2018/01/26/changes-leg-ulcer-managementaffecting/ [Accessed 26 May 2018].

2 Hopkins A. Venousnewscom, 2018. Available: https://venousnews. $\mathrm{com} /$ changing-the-narrative-around-light-compression/. [Accessed 02 Aug 2019].

3 Maessen-Visch MB, de Roos K-P. Dutch venous ulcer guideline update. Phlebology 2014;29:153-6.

4 Andriessen A, Apelqvist J, Mosti G, et al. Compression therapy for venous leg ulcers: risk factors for adverse events and complications, contraindications - a review of present guidelines. J Eur Acad Dermatol Venereol 2017;31:1562-8.

5 Kelechi TJ, Johnson JJ, WOCN Society. Guideline for the management of wounds in patients with lower-extremity venous disease: an executive summary. $J$ Wound Ostomy Continence Nurs 2012;39:598-606.

6 Hopkins A, Bull R, Worboys F. Needing more: the case for extra high compression for tall men in UK leg ulcer management. Veins and Lymphatics 2017;6.

7 Partsch H, Mortimer P. Compression for leg wounds. Br J Dermatol 2015;173:359-69.

8 Berwick DM, Kelley E, Kruk ME, et al. Three global health-care quality reports in 2018. Lancet 2018;392:194-5.
9 Lilford RJ. Implementation science at the crossroads. BMJ Qual Saf 2018;27:331-2

10 Braithwaite J. Changing how we think about healthcare improvement. BMJ 2018;361:k2014.

11 Samuriwo R. Wounds research network (WReN) - a community of practice for improving wound care-related trials. J European Wound Manag Assoc 2019;20:39-42.

12 Allen D. Institutionalising emergent organisation in health and social care. J Health Organ Manag 2019;33:764-75.

13 Plsek PE, Greenhalgh T. Complexity science: the challenge of complexity in health care. BMJ 2001;323:625-8.

14 Pattison S, Samuriwo R. Ceeing compassion in care: more than 'Six C'S'? Nurs Philos 2016;17:140-3.

15 Braithwaite J. Changing how we think about healthcare improvement. BMJ 2018;361:k2014-k.

16 Martin GP, Dixon-Woods M. Can we tell whether hospital care is safe? Br J Hosp Med 2014;75:484-5.

17 Pronovost PJ, Marsteller JA. Creating a fractal-based quality management infrastructure. $J$ Health Organ Manag 2014;28:576-86.

18 Braithwaite J, Churruca K, Ellis LA, et al. Complexity science in healthcare. aspirations, approaches and accomplishements. A white paper. Sydney, Australia: Australian Institute of Health Innovation, Macquarie University, Sydney, Australia, 2017.

19 Peters MDJ, Godfrey C, Mclnerney P, et al. Chapter 11: Scoping Reviews. In: Aromataris E, Munn Z, eds. Joanna Briggs Institute reviewer's manual. Adelaide, Australia: The Joanna Briggs Institute, 2017. https://reviewersmanual.joannabriggs.org/

20 Tricco AC, Lillie E, Zarin W, et al. PRISMA extension for scoping reviews (PRISMA-ScR): checklist and explanation. Ann Intern Med 2018;169:467-73.

21 Peters MDJ, Godfrey C, Mclnerney P, et al. Chapter 11: Scoping Reviews. In: Aromataris E, Munn Z, eds. Joanna Briggs Institute reviewer's manual. The Joanna Briggs Institute, 2017. https:// reviewersmanual.joannabriggs.org/

22 Ouzzani M, Hammady H, Fedorowicz Z, et al. Rayyan-a web and mobile APP for systematic reviews. Syst Rev 2016;5:210.

23 Moffatt CJ, Franks PJ, Oldroyd M, et al. Community clinics for leg ulcers and impact on healing. BMJ 1992;305:1389-92.

24 McInnes E, Cullum N, Nelson A, et al. RCN guideline on the management of leg ulcers. Nurs Stand 1998;13:61-3.

25 Cullum N, Fletcher A, Semlyen A, et al. Compression therapy for venous leg ulcers. Qual Health Care 1997;6:226-31.

26 Williams C. The management of patients with venous leg ulcers: new guidelines. Br J Nurs 1999;8:489-98.

27 Bennett B, Provost L. What's your theory? driver diagram serves as tool for building and testing theories for improvement organizational improvement, 2015: 36-43.

28 Langley GJ, Moen RD, Nolan KM, et al. The improvement guide: a practical approach to enhancing organizational performance. 2 edn. San Francisco: Josey-Bass, 2009. 